\title{
Análise dos critérios diagnósticos dos distúrbios do metabolismo de glicose e variáveis associadas à resistência insulínica
}

\author{
Diagnostic criteria of the glucose metabolism disorders and variables associated to insulin resistence
}

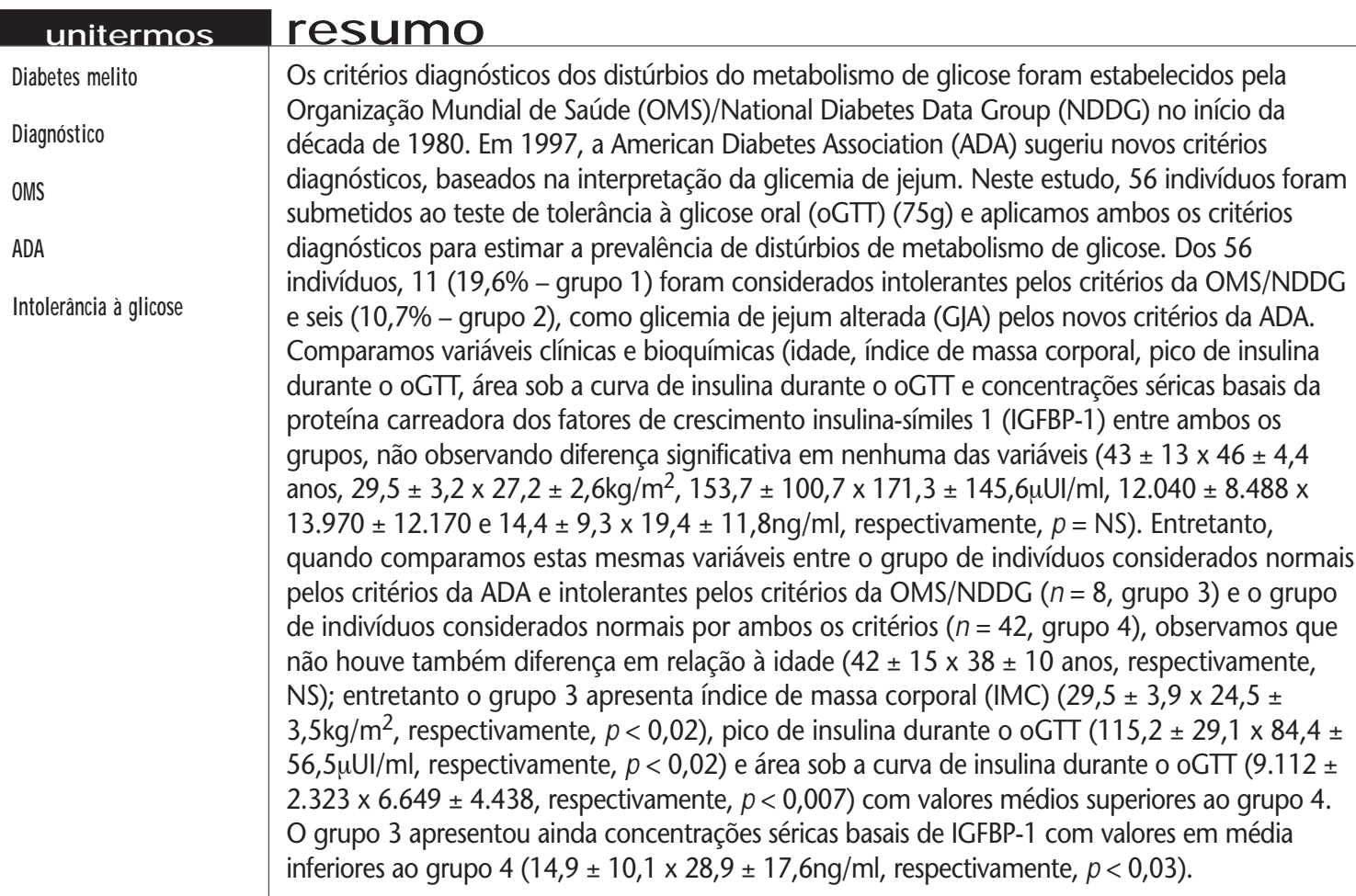

\section{abstract}

The diagnostic criteria of the glucose metabolism disorders had been established by the World Health Organization (WHO)/National Data Group (NDDG) in the beginning of the 1980's. In

1997, the American Diabetes Association (ADA) suggested new criteria, based upon the interpretation of the fasting glucose. In this study, 56 subjects underwent an oral glucose tolerance test (oGTT), and we have applied both criteria to estimate the prevalence of the glucose metabolism disorders. Out of 56 subjects, 11 (19,6\% - Group 1) had been considered glucose intolerants by the criteria of the WHO/NDDG and 6 (10,7\% - Group 2) as impaired fasting glycemia (IFG) by the ADA

new criteria. We have compared clinical and biochemical variables (age, BMI, insulin peak during

OGTT, area under the insulin curve during $0 \mathrm{GTT}$ and serum concentrations of IGFBP-1) between both groups, not observing differences in any of the variables ( $43 \pm 13 \times 46 \pm 4.4$ years, $29.5 \pm 3.2$ $\times 27.2 \pm 2.6 \mathrm{~kg} / \mathrm{m}^{2}, 153.7 \pm 100.7 \times 171.3 \pm 145.6 \mathrm{ul} / \mathrm{ml}, 12,040 \pm 8,488 \times 13,970 \pm 12,170 \mathrm{e}$

$14.4 \pm 9.3 \times 19.4 \pm 11.8 \mathrm{ng} / \mathrm{ml}$, respectively, $p=N S)$. However, when we compare these same variables between the subjects considered normal by the ADA criteria and intolerants by the WHO/ NDDG ( $n=8$, Group 3) and the subjects considered normal by both criteria ( $n=42$, Group 4), we observed that it also did not have difference in relation to age $(42 \pm 15 \times 38 \pm 10$ years,

respectively, NS), however, Group 3 presents BMI $\left(29.5 \pm 3.9 \times 24.5 \pm 3.5 \mathrm{~kg} / \mathrm{m}^{2}\right.$, respectively, $\mathrm{p}<0.02)$, insulin peak $(115.2 \pm 29.1 \times 84.4 \pm 56.5 \mu \mathrm{Ul} / \mathrm{ml}$, respectively, $\mathrm{p}<0.02)$ and area under the insulin curve $(9,112 \pm 2,323 \times 6,649 \pm 4,438$, respectively, $p<0.007)$ with higher values than

Group 4. Group 3 still presented serum concentration of IGFBP-1 with lower values than Group 4 $(14.9 \pm 10.1 \times 28.9 \pm 17.6 \mathrm{ng} / \mathrm{ml}$, respectively, $\mathrm{p}<0.03)$. key words

Diabetes mellitus

Diagnostic criteria

WHO

$A D A$

Impaired glucose tolerance
Divisão de Endocrinologia e Metabologia, Departamento de Clínica Médica da Faculdade de Medicina de Ribeirão Preto da Universidade de São Paulo (USP). 


\section{Introdução}

Os critérios clássicos para o diagnóstico do diabetes melito (DM) anteriormente adotados $(1,2)$ eram baseados na obtenção de glicemia em sangue venoso em amostra randômica (independente de jejum) igual ou superior a $200 \mathrm{mg} / \mathrm{dl}$ ou duas glicemias de jejum, em dias diferentes, iguais ou superiores a $140 \mathrm{mg} / \mathrm{dl}$, associadas aos sintomas da doença. Se determinado indivíduo apresentasse glicemia de jejum igual ou superior a $110 \mathrm{mg} / \mathrm{dl}$ e inferior a $140 \mathrm{mg} / \mathrm{dl}$, estaria indicada a realização do teste de tolerância à glicose oral (oGTT) para estabelecimento do diagnóstico definitivo, cuja interpretação é baseada em critérios estabelecidos pelo National Diabetes Data Group (NDDG) e pela Organização Mundial de Saúde (OMS). Segundo a OMS, o diagnóstico de DM é confirmado na observação de glicemia basal (jejum) igual ou superior a $140 \mathrm{mg} / \mathrm{dl}$ ou glicemia 120 minutos após a sobrecarga oral de $75 \mathrm{~g}$ de glicose igual ou superior a $200 \mathrm{mg} /$ dl. Segundo o NDDG, o diagnóstico era estabelecido na presença de uma glicemia igual ou superior a $200 \mathrm{mg} / \mathrm{dl}$ em quaisquer tempos (30, 60 ou 90 minutos) após a sobrecarga oral de glicose associada a glicemia igual ou superior a $200 \mathrm{mg} / \mathrm{dl}$ no tempo 120 minutos. O diagnóstico da intolerância a glicose (IG) seria estabelecido com a obtenção de uma glicemia igual ou superior a $200 \mathrm{mg} / \mathrm{dl}$ em quaisquer tempos (30, 60 ou 90 minutos) após a sobrecarga de glicose associada a glicemia entre $140 \mathrm{mg} / \mathrm{dl}$ e $199 \mathrm{mg} / \mathrm{dl}$ no tempo de 120 minutos.

No final de 1997, a American Diabetes Association (ADA) sugeriu como critério diagnóstico do DM, baseada em critérios de sensibilidade e especificidade diagnóstica, a obtenção de apenas uma glicemia de jejum igual ou superior a $126 \mathrm{mg} / \mathrm{dl}$ (3). Sugeriu ainda a não-utilização do oGTT como método diagnóstico em rotina clínica, criando uma nova entidade nosológica denominada glicemia de jejum alterada (GJA) para o intervalo de glicemia entre $111 \mathrm{mg} / \mathrm{dl}$ e $125 \mathrm{mg} / \mathrm{dl}$. Este critério diagnóstico da ADA ainda não foi amplamente aceito, havendo controvérsias na sua adoção.

O objetivo do presente estudo é estimar a prevalência de distúrbios do metabolismo de carboidratos em uma população de indivíduos e comparar variáveis clínicas (idade e índice de massa corporal - IMC) e bioquímicas (pico de insulina durante oGTT, área sob curva de insulina durante oGTT e concentrações séricas basais de proteína carreadora dos fatores de crescimento insulina-símile 1 IGFBP-1), associadas à resistência insulínica, entre população de indivíduos com diagnóstico de normalidade, IG ou GJA de acordo com estes diferentes critérios.

\section{Material e métodos}

\section{População estudada}

Foram estudados 56 indivíduos adultos de ambos os sexos (30 F), com idade variando entre 21 e 74 anos (média \pm DP, 40,1 $\pm 11,7$ anos). Estes indivíduos não apresentavam quaisquer evidências clínicas de desnutrição, doença hepática ou renal, endocrinopatias ou outras doenças agudas ou crônicas por ocasião do estudo, além de não utilizarem quaisquer tipos de medicação.

O estudo foi aprovado pelo Comitê de Ética local (HCFMRP/USP) e todos os indivíduos foram orientados detalhadamente quanto à metodologia e às finalidades do estudo e concordaram voluntariamente em participar

Em todos os indivíduos foram verificados peso e estatura para determinação do IMC.

\section{OGTT}

Após jejum noturno de 12 horas, todos os indivíduos foram submetidos ao oGTT, utilizando-se $75 \mathrm{~g}$ de glicose diluída em $250 \mathrm{ml}$ de água. Após 30 minutos de repouso, amostras de sangue de veia periférica de antebraço foram obtidas nos tempos -15min e 0min, para determinação de glicemia, insulinemia e concentração sérica basal de IGFBP-1, após o qual os indivíduos ingeriram a solução de glicose, e novas amostras de sangue foram obtidas nos tempos $+30 \mathrm{~min}$ +60 min +90 min e +120 min, para determinação das concentrações plasmáticas de glicose e insulina. No final de cada prova, as amostras foram centrifugadas e determinaram-se imediatamente as glicemias, sendo o restante congelado até a realização dos ensaios da insulina e IGFBP-1.

\section{Determinação da glicemia e da insulina plasmática}

As determinações de glicemia, insulina e IGFBP-1 foram realizadas em duplicata, sendo que amostras de um mesmo indivíduo foram determinadas em um mesmo ensaio. A determinação de glicemia foi realizada através do método da glicose-oxidase, em aparelho automatizado Glucose Analyser 2 (Beckman Instruments, Inc., Fullerton, Ca, EUA).

A determinação das concentrações plasmáticas de insulina foi realizada utilizando-se kits Coat-a-Count ${ }^{\circledR}$ da Diagnostic Products Corporation (DPC - Los Angeles, Ca, EUA) (4). Trata-se de um radioimunoensaio de duplo anticorpo e fase sólida, com o primeiro anticorpo policlonal adsorvido à superfície de tubos de polipropileno. A curva padrão foi constituída com as concentrações de $5 \mathrm{uUl} / \mathrm{ml}, 15 \mathrm{uUl} / \mathrm{ml}, 50 \mathrm{uUl} / \mathrm{ml}$ $100 \mu \mathrm{UI} / \mathrm{ml}, 200 \mu \mathrm{UI} / \mathrm{ml}$ e $400 \mu \mathrm{UI} / \mathrm{ml}$. O ensaio necessita de uma incubação overnight, e a leitura é realizada em contador 
gama (ANSR - Abbott Laboratories). Todos os reagentes são fornecidos pelo fabricante. Os resultados são expressos em $\mu \mathrm{UI} / \mathrm{ml}$ e a sensibilidade do método é de $1,2 \mu \mathrm{UI} / \mathrm{ml}$. Os coeficientes de variação entre/intra-ensaios foram, respectivamente, de $8 \%$ e $3,5 \%$, na dose $\mathrm{B} / \mathrm{Bo}=0,5$.

\section{Determinação das concentrações séricas basais de IGFBP-1}

A determinação das concentrações séricas basais de IGFBP-1 foi realizada utilizando-se kits Active ${ }^{\mathrm{TM}}$ Total IGFBP-1, da Diagnostic Systems Laboratories (DSL - Webster, Texas, EUA). Trata-se de um ensaio imunorradiométrico de duplo anticorpo e fase sólida que utiliza a técnica do sanduíche. O primeiro anticorpo monoclonal anti-IGFBP-1 encontra-se adsorvido à superfície de tubos e o segundo anticorpo policlonal é marcado com 125I. A curva padrão foi constituída com as seguintes concentrações: $0,5 \mathrm{ng} /$ $\mathrm{ml} ; 2 \mathrm{ng} / \mathrm{ml} ; 10 \mathrm{ng} / \mathrm{ml} ; 40 \mathrm{ng} / \mathrm{ml} ; 80 \mathrm{ng} / \mathrm{ml}$ e $160 \mathrm{ng} / \mathrm{ml}$. O ensaio necessita de uma incubação sob agitação rotatória de cerca de 15 horas, sendo a leitura realizada em contador gama (ANSR - Abbott Laboratories). Todos os reagentes são fornecidos pelo fabricante (5). Os resultados são expressos em ng/ml e a sensibilidade do método é de $0,33 \mathrm{ng} / \mathrm{ml}$. Os coeficientes de variação entre/intra-ensaios foram, respectivamente, de $12 \%$ e $4 \%$ no ponto médio da curva padrão.

\section{Análise dos resultados}

A glicemia de jejum e como a concentração sérica basal de IGFBP-1 foram consideradas como a média dos valores obtidos nos tempos $-15 \mathrm{~min}$ e $0 \mathrm{~min}$ do oGTT. Foram comparados idade, IMC, pico de insulina durante o oGTT, área sob a curva (ASC) de insulina durante o oGT e concentração sérica basal de IGFBP-1 entre os diversos grupos, separados de acordo com os diferentes critérios diagnósticos. Os resultados são expressos como média e desvio padrão da média. Para análise estatística foram utilizados os testes nãoparamétricos de Wilcoxon-Mann-Whitney e de Sperman, adotando-se o nível de significância de 5\%. A análise estatística foi realizada utilizando-se o programa Graph Pad Prism ${ }^{\text {TM }}$, da indústria Graph Pad Software (San Diego, Ca, EUA).

\section{Resultados}

\section{Correlação entre glicemia de jejum e glicemia 120min pós-glicose}

Observamos correlação positiva e significativa entre glicemia de jejum e glicemia $120 \mathrm{~min}$ pós-glicose na população estudada $(n=56 ; r=0,55 ; p<0,002)$ (Figura 1 ).

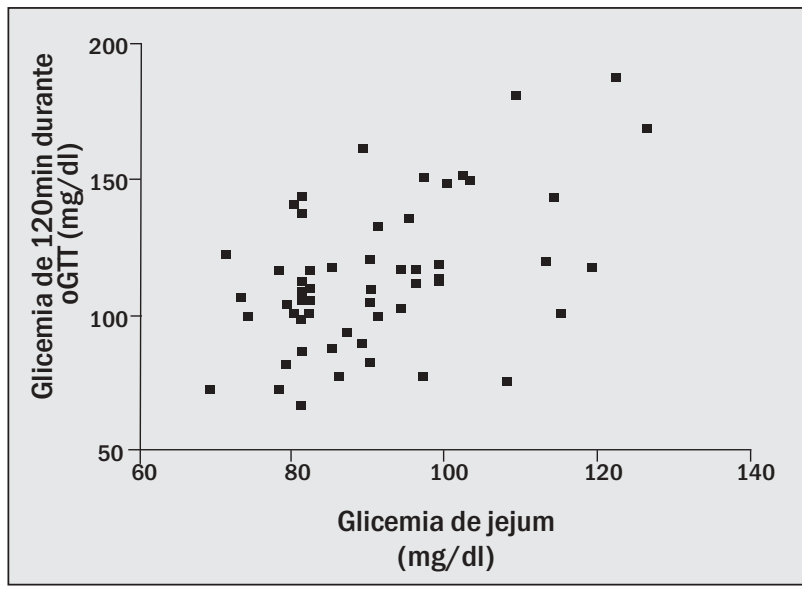

Figura 1 - Correlação entre glicemia de jejum e glicemia de 120 minutos durante 0 oGTT em 56 indivíduos $(r=0,48 ; p<0,001)$

\section{Diagnóstico de normalidade, IG, GJA e DM, de acordo com os diferentes critérios, na população estudada}

Não observamos nenhum indivíduo com diagnóstico de DM com quaisquer dos critérios utilizados. Dos 56 indivíduos, 11 (19,6\%) foram classificados como IG pelos critérios da OMS/NDDG e seis $(10,7 \%)$, como GJA pelo critério da ADA. Houve concordância entre ambos os critérios quanto ao diagnóstico de intolerância em apenas três pacientes, e também apenas três indivíduos considerados normais pelos critérios do OMS/NDDG (total de 45) foram considerados alterados pelo critério da ADA. Dos indivíduos considerados normais pelos critérios da ADA (total de 50), oito foram considerados intolerantes pelo critério da OMS/NDDG.

\section{Comparação das variáveis associadas à resistência insulínica entre indivíduos considerados IG pelos critérios da OMS/ NDDG (grupo 1) e GJA pela ADA (grupo 2)}

Com base nos critérios da OMS/NDDG, 45 indivíduos foram considerados normais e 11 indivíduos (19,6\% grupo 1), IG. Com base nos critérios da ADA, 50 indivíduos foram considerados normais e seis indivíduos (10,7\% -grupo 2), GJA. Não observamos diferença estatisticamente significativa entre os grupos 1 e 2 (Figura 2) em relação a idade (43 $\pm 13 \times 46,0 \pm 4,4$ anos, respectivamente, $\mathrm{NS})$, IMC $\left(29,5 \pm 3,2 \times 27,2 \pm 2,6 \mathrm{~kg} / \mathrm{m}^{2}\right.$, respectivamente, NS), pico de insulina durante o oGTT $(153,7 \pm 100,7 \times$ $171,3 \pm 145,6 \mu \mathrm{Ul} / \mathrm{ml}$, respectivamente, NS), ASC insulina durante oGTT $(12.040 \pm 8.488 \times 13.970 \pm 12.170$, respectivamente, NS) e concentrações séricas basais de IGFBP$1(14,4 \pm 9,3 \times 19,4 \pm 11,8$, respectivamente, NS). 

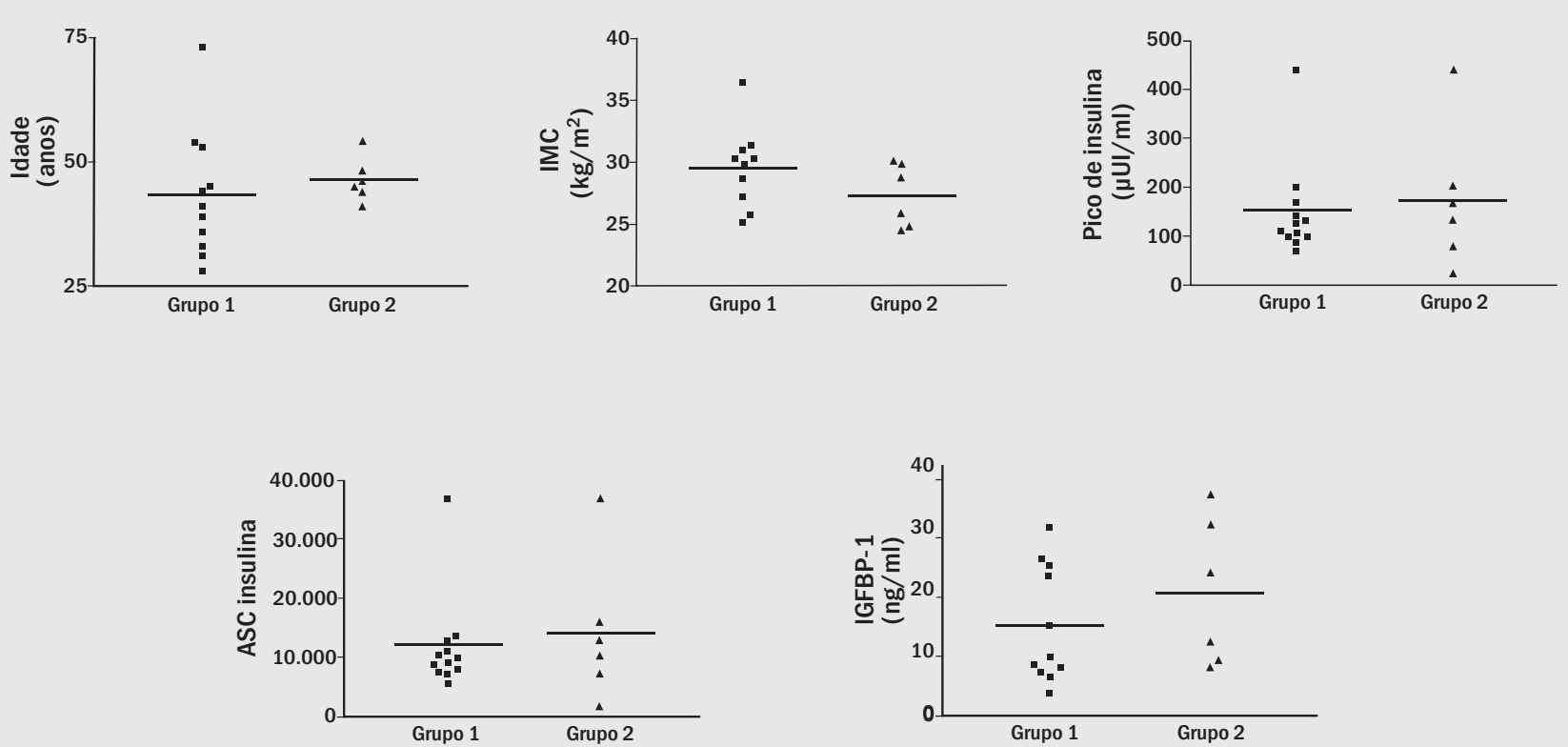

Figura 2 - Comparação entre idade (anos), IMC ( $\left.\mathrm{kg} / \mathrm{m}^{2}\right)$, pico de insulina durante o oGT ( $\left.\mathrm{UU} / \mathrm{lml}\right)$, área sob a curva de insulina durante 0 oGTT e concentraç̃oes séricas basais de IGFBP-1 (ng/ml) entre grupos de indivíduos intolerantes à glicose segundo critérios da OMS/NDDG (grupo $1, \mathrm{n}=11$ ) e com glicemia de jejum alterada segundo critérios da ADA (grupo $2, n=6$ ) [p = NS em todas as comparações]

\section{Comparação das variáveis associadas à resistência insulínica entre indivíduos considerados normais pelos critérios da ADA e IG pelos critérios da OMS/ NDDG (grupo 3) e indivíduos considerados normais pelos critérios da ADA e da OMS/ NDDG (grupo 4)}

Oito indivíduos foram considerados normais pelos critérios da ADA e IG pelos critérios da OMS/NDDG (grupo 3). Comparamos as mesmas variáveis entre estes indivíduos e os indivíduos considerados normais pelos critérios da ADA e da OMS/NDDG (grupo 4, n=42). Os indivíduos do grupo 3 apresentaram idade semelhante ao grupo 4 (42 $\pm 15 \times 38 \pm 10$ anos, respectivamente, NS), entretanto apresentaram IMC $\left(29,5 \pm 3,9 \times 24,5 \pm 3,5 \mathrm{~kg} / \mathrm{m}^{2}\right.$, respectivamente, $\mathrm{p}<0,02)$, pico de insulina durante o oGTT $(115,2 \pm 29,1 \times 84,4 \pm 56,5 \mu \mathrm{Ul} / \mathrm{ml}$, respectivamente, $p<$ $0,02)$ e ASC insulina durante o oGTT $(9.112 \pm 2.323 x$ $6.649 \pm 4.438$, respectivamente, $p<0,007$ ) com valores em média superiores aos do grupo 3. Apresentaram ainda concentrações séricas basais de IGFBP-1 $(14,9 \pm 10,1 \times$ $28,9 \pm 17,6$, respectivamente, $p<0,03$ ) com valores em média inferiores ao grupo 4 (Figura 3 ).

\section{Discussão}

No início do século XX, o diagnóstico do diabetes melito era baseado na observação dos sintomas clássicos da doença (polifagia, poliúria e polidipsia) associados à presença de substâncias redutoras na urina. Com a disponibilidade de ensaios para determinação da glicemia, a presença de hiper- glicemia de jejum passou a ser condição sine qua non para a confirmação diagnóstica (6). O oGTT foi introduzido como ferramenta de pesquisa nos anos 1920 (7) e, nos anos 1950 e 1960, com ênfase no diagnóstico e intervenção precoces, passou a ser largamente utilizado em todo o mundo no diagnóstico do DM. O atual critério de interpretação do oGTT foi sugerido pelo NDDG em 1979 (1) e validado pela OMS em 1980 (2), com modificações em 1985 (8) e 1994 (9). Os cutoffs sugeridos foram baseados em estudos epidemiológicos longitudinais (10-16) e correlacionados com a incidência de complicações crônicas da doença.

Em 1997, o Expert Committee on the Diagnosis and Classification of Diabetes Mellitus da American Diabetes Association (ADA) recomendou novos critérios clínicos para o diagnóstico do $\mathrm{DM}$, baseados apenas na glicemia de jejum (3). Os argumentos sugeridos pela ADA para a adoção destes novos critérios são baseados na observação epidemiológica, com destaque no número de pacientes não-diagnosticados, e na forte correlação positiva entre a glicemia de jejum e a glicemia de $120 \mathrm{~min}$ após a sobrecarga oral de glicose e o fato de a glicemia de jejum ser altamente reprodutível e disponível em todo o mundo. Critica ainda as limitações do oGTT, um teste não-fisiológico que demanda tempo, desconforto, custo e ainda apresenta baixa reprodutibilidade. Sugere, ainda, uma nova entidade nosológica, denominada GJA, atribuída a indivíduos que apresentam glicemia de jejum entre $111 \mathrm{mg} / \mathrm{dl}$ e $125 \mathrm{mg} / \mathrm{dl}$.

Durante as últimas décadas uma série de conhecimentos sobre a fisiopatologia dos distúrbios do metabolismo 

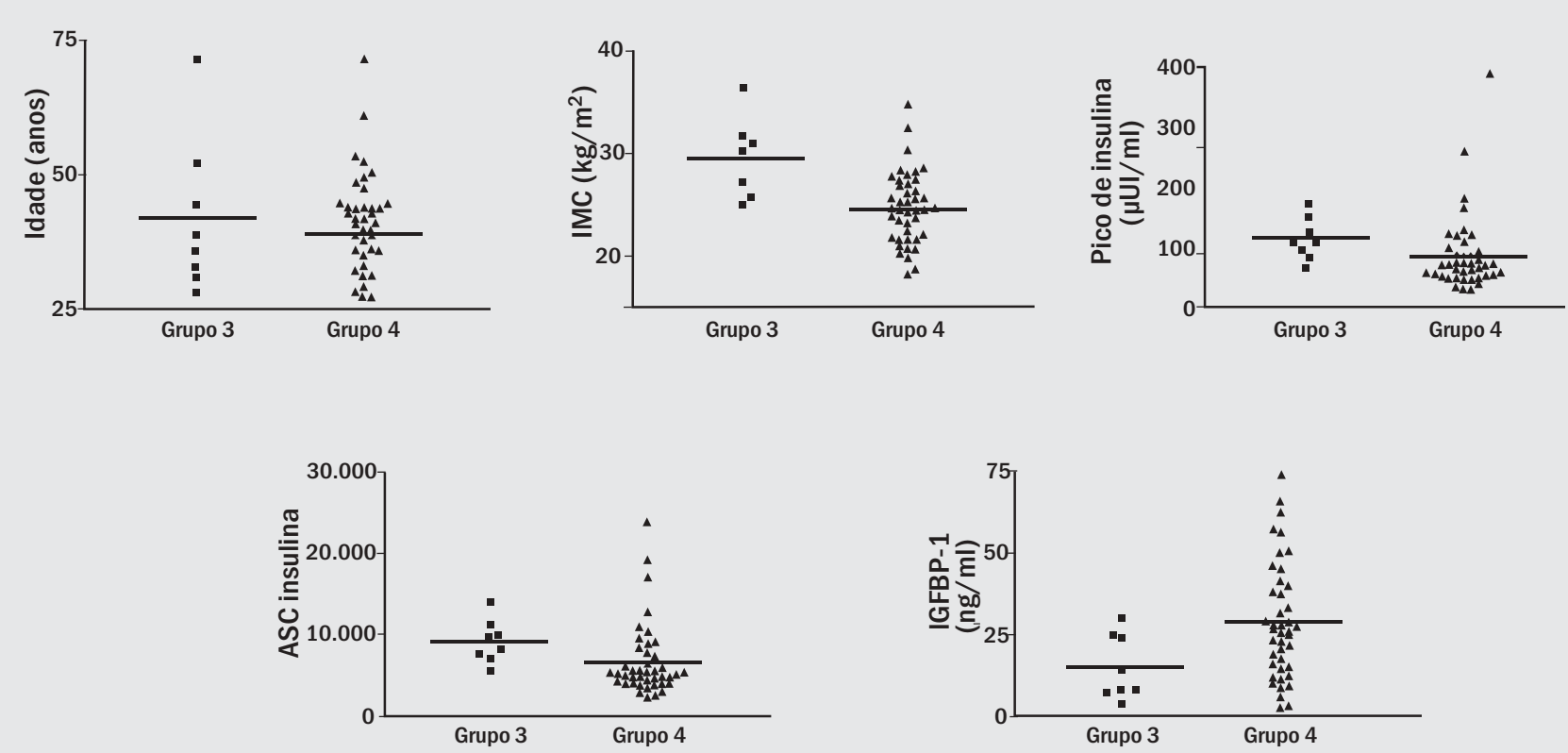

Figura 3 - Comparação entre idade (anos), IMC ( $\left.\mathrm{kg} / \mathrm{m}^{2}\right)$, pico de insulina durante o oGT $(\mu \mathrm{Ul} / \mathrm{ml})$, área sob a curva de insulina durante o oGTT e concentrações séricas basais de IGFBP-1 (ng/mL) entre grupos de indivíduos normais pelos critérios da ADA e intolerantes pelos critérios da OMS/NDDG (grupo $3, \mathrm{n}=8$ ) e indivíduos considerados normais pelos critérios da ADA e da OMS/NDDG (grupo $4, n=42$ )

de glicose, com destaque para os mecanismos de resistência insulínica no DM do tipo 2, foi adquirida. Variáveis clínicas e bioquímicas como idade $(17,18)$, obesidade $(19,20$, $21,22,23)$ e hiperinsulinemia $(24,25,26,27)$ estão claramente associadas ao DM do tipo 2 e à intolerância a glicose. Na década de 1980 foi isolada uma proteína carreadora dos fatores de crescimento insulina-símile, de baixo peso molecular e GH independente, denominada IGFBP-1 (28). Experimentos posteriores demonstraram uma clara regulação negativa das concentrações séricas desta proteína pela insulina, isto é, as concentrações séricas de IGFBP-1 apresentam correlação negativa com as concentrações plasmáticas de insulina. Observou-se ainda que pacientes com DM e hiperinsulinemia apresentam concentrações séricas basais de IGFBP-1 com valores em média inferiores aos dos indivíduos normais (29-31).

Neste estudo, 56 indivíduos foram submetidos ao oGTT e classificados como normais ou intolerantes com base nos critérios clássicos da OMS/NDDG e nos novos critérios sugeridos pela $\mathrm{ADA}$. A aparente discrepância diagnóstica entre ambos os critérios observada neste estudo já foi observada em outros estudos epidemiológicos (32-37). Os dados sugerem que a utilização da glicemia de jejum apresenta limitações no diagnóstico da intolerância a carboidratos, uma condição clínica de importância prognóstica.

Comparamos ainda, entre os indivíduos considerados IG pelos critérios do OMS/NDDG $(n=11)$ e os considerados GJA pelos critérios da $\operatorname{ADA}(n=6)$, variáveis associadas à resistência insulínica. Observamos que não houve diferença estatística sig- nificativa entre os dois grupos em relação a idade, IMC, insulinemia e concentrações séricas basais de IGFBP-1. Estes dados sugerem que ambos os critérios identificaram uma população semelhante em relação à resistência insulínica. No entanto, quando comparamos estas mesmas variáveis entre o grupo de indivíduos considerados normais pelo critério da ADA e intolerantes pelos critérios da OMS/NDDG (grupo $3, n=8$ ) e o grupo de indivíduos considerados normais por ambos os critérios (grupo 4, $n=42$ ), observamos que, apesar de não haver diferença significativa em relação à idade, o grupo 3 apresentou IMC e insulinemia com valores em média superiores aos do grupo 4. O grupo 3 apresentou, ainda, concentrações séricas basais de IGFBP-1 com valores em média inferiores aos do grupo 4. Estes dados demonstram que o grupo 3 apresenta maior resistência insulínica e hiperinsulinemia, sendo mais bem identificado pelos critérios da OMS/NDDG. No entanto, observando-se de outro ponto de vista, poderíamos atribuir esta diferença ao fato de que a utilização dos critérios da ADA identifica mais precocemente indivíduos com intolerância a carboidratos, em uma fase mais inicial de resistência insulínica. Apesar de improvável, apenas um estudo longitudinal seria capaz de responder esta questão.

Em resumo, uma questão importante sugerida por este e por outros estudos e ainda não respondida: os novos critérios diagnósticos sugeridos pela ADA e a criação de uma nova entidade nosológica denominada GJA identificam o mesmo grupo de indivíduos com IG clássica, diagnosticada pelos critérios da OMS/NDDG, sabidamente expostos a maior risco de desenvolvimento de DM e doença cardiovascular? 


\section{Referências}

1. N ational D iabetes Data Group. Classification and diagnosis of diabetes mellitus and other categories of glucose intolerance. Diabetes, 28: 1039-57, 1979.

2. World Health 0 rganization Expert Committee on Diabetes M ellitus. Second report. G eneva, W orld Health Organization, 1980 (Tech. Rep. Serv., n. 646).

3. Expert Committee on the Diagnosis and Classification of D iabetes Mellitus. Report of the Expert Committee on the $D$ iagnosis and Classification of $D$ iabetes M ellitus. Diabetes Care, 20:1183-97, 1997.

4. Coat-a-count Insulin. D iagnostic Products Corporation, Los Angeles, Ca. Manual de instruções, 1997.

5. Active Total IGFBP-1. Diagnostic System Laboratories Inc., W ebster,Texas. Manual de Instruções, 1996.

6.Bennett,P.H.The diagnosis of diabetes.N ew international classification and diagnostic criteria. Ann. Rev. Med., 34:295-309, 1983.

7. John, H.J. G lucose tolerance and its value in diagnosis. J. M etab. Research, 1:497-548, 1922.

8.W orld Health O rganization Study Group on D iabetes Mellitus.Geneva, W orld Health Organization, 1985 (Tech. Rep. Serv.n. 727).

9.Prevention ofDiabetes Mellitus.Report ofW HO Study Group.Geneva, W orld Health Organization, 1994 (Tech. Rep. Serv.n. 844).

10. Rushforth, N .B. et al. Diabetes in the Pima Indians: evidence of bimodality in glucose tolerance distributions. Diabetes, 20: 756-765, 1971.

11. Rushforth, N.B, et al. Comparison of the two- and one-hour glucose levels of the oral GTT in the diagnosis of diabetes in Pima Indians. Diabetes, 24: 538-46, 1975.

12. Rosenthal, M.; McMahan, C.A.\& Stern, M.P. Evidence of bimodality of two-hour plasma glucose concentrations in Mexican-A mericans: results from the San Antonio Heart Study. J. Chron. Dis., 38: 5-16, 1985.

13. Zimmet, P. \& W hitehouse, S. Bimodality of fasting and twohour glucose tolerance distributed in a Micronesian population. Diabetes, 27: 793-800, 1978.

14. Raper, L.R. et al. Bimodality in glucose tolerance distributions in the urban Polynesian population of W estern Samoa. Diabetes Res., 1: 19-26, 1984.

15.Tillil, H . et al. Bimodal distribution of the 2-hour blood glucose value during O GTT among first-degree relatives of type 2-diabetics in a C aucasoid population. Diabetes Res. Clin. Pract. (Suppl 1), S560, 1985.

16. Rushforth, N .B.; Miller, M. \& Bennett, P.H. Fasting and two-hour post-load glucose levels for the diagnosis of the diabetes.The relationship between glucose levels and complications of diabetes in the Pima Indians. Diabetologia, 16: 373-9, 1979.

17. Fink, R.I. et al. Mechanisms of insulin resistance in aging. J. Clin. Invest., 71: 1523-35, 1983.

18. Rowe, S.W . et al. Characterization of the insulin resistance in aging. J. Clin. Invest., 71: 1581-7, 1983.

19. Rabinowitz, D. \& Zieler, K.L. Forearm metabolism in obesity and its response to intra-arterial insulin: characterization of insulin resistance and evidence for adaptative hyperinsulinism. J. Clin. Invest., 41:2173-81, 1962.

20. 0 lefsky, J.M.; Reaven, G .M . \& Farquhar, J.W . Effects of weight reduction on obesity: studies of carbohydrate and lipid metabo lism. J. Clin. Invest., 53:64-76, 1974.

21. Kissebah, A.H. et al. Relation of body fat distribution to metabolic complications of obesity...Clin.Endocrinol.M etab.,54:254-60,1982.
22. Bogardos, C. et al. Relationship between degree of obesity and in vivo insulin action in man. Am. J. Physiol., 248:E286-91, 1985.

23. Zimmet, P.Z. Kelly W est Lecture 1991 - Challenges in diabetes epidemiology - from west to the rest. Diabetes Care, 15: 232-52, 1992.

24.Turner, R.C . et al.Insulin deficiency and insulin resistance interaction in diabetes: estimation of their relative contribution by feedback analysis from basal plasma insulin and glucose concentrations. Metabolism, 28: 1086-96, 1979.

25. Kolterman, O.G. et al. Receptor and postreceptor defects contribute to the insulin resistance in noninsulin-dependent diabetes mellitus. J. Clin. Invest., 68: 957-69, 1981.

26.D eFronzo, R.A.Lilly Lecture 1987.The triumvirate:b-cell, muscle,liver: a collusion responsible for N ID D M. Diabetes, 37:667-87, 1988.

27. Reaven, G.M. Banting Lecture 1988: Role of insulin resistance in human disease. Diabetes, 37: 1595-1607, 1988.

28. Póvoa, G.; Roovete, A. \& Hall, K. Cross-reaction of serum somatomedin-binding protein in a radio immunoassay developed for somatomedin-binding protein isolated from human amniotic fluid. Acta Endocrinol. (Copenh), 107:563-70, 1984.

29. H all, K. et al. Serum level of somatomedins and somatomedinbinding protein in pregnant women with type 1 or gestational diabetes and their infants. J. Clin. Endocrinol. M etab., 63:1300-6, 1986.

30. Hall, K.; Lunding, G. \& Póvoa, G. Serum levels of the low molecular weight form of insulin-like growth factor-binding protein in healthy subjects and patients with growth hormone deficiency, acromegaly and anorexia nervosa. Acta Endocrinol. (Copenh), 118: 321-6, 1988.

31.Holly,J.M.P.et al.C ircadian variation of GH-independent IG F-binding protein in diabetes mellitus and its relationship to insulin. A new role for insulin? Clin. Endocrinol. (0xf), 29:667-75, 1988.

32. Stolk, R.P.; 0 rchard, T.J. \& Grobbee, D.E. W hy use the oral tolerance test? Diabetes Care, 18: 1045-9, 1995.

33. McCance, D.R. et al. Diagnosing diabetes mellitus - Do we need new criteria? Diabetologia, 40:247-55, 1997.

34. The DECODE-study group on behalf of the European $D$ iabetes Epidemiology G roup. Is fasting gluco se sufficient to define diabetes? Epidemiological data from 20 European studies. Diabetologia, 42:647-54, 1999.

35. Lee, E.T. et al. Prevalence of undiagnosed diabetes in three A merican Indians populations. A comparison of the 1997 A merican Diabetes A sso ciation diagnostic criteria and the 1985 W orld Health O rganization diagnostic criteria: the strong heart study. Diabetes Care, 23: 181-6, 2000.

36. Albert, K.G.G.M. \& Zimmet, P. N ew diagnostic criteria and classification of diabetes - again? (Editorial) Diabet. Med. 15: 535-6, 1998.

37. Gimeno, S.G.A. et al.The Japanese-Brazilian D iabetes Study Group. Comparison of glucose tolerance categories according to World Health 0 rganization and American D iabetes Association diagnostic criteria in a populationbased study in Brazil. Diabetes Care, 21: 1889-92, 1998.

Endereco para correspondência

Augusto C. F. Costa

Área de Clínica Médica

Faculdade de Medicina/UnB

CEP 70919-970 - Brasilia-DF 